\title{
The Frequency of Intranasal Synechiae Formation between Nasal Septum and Lateral Nasal Wall after Endoscopic Sinus Surgery Among Patients with Sinonasal Polyposis
}

\section{Damish Arsalan, ${ }^{1}$ Sarwat Hassan Syed, ${ }^{2}$ Ghulam Murtaza, ${ }^{3}$ Mohammad Qamar Nasir, ${ }^{4}$ Muhammad Awais Amin, ${ }^{5}$ Aamer Ayub Awan ${ }^{6}$}

\begin{abstract}
Objectives: To determine the frequency of intranasal synechiae formation between lateral nasal wall and nasal septum after (ESS) Endoscopic Sinus Surgery among patients with Sino nasal Polyposis

Methods: After obtaining approval from the ethical committee of our Hospital, a total number of 150 subjects fulfilling the study criteria were recruited in the research which was conducted in the department of Otorhinolaryngology, Services Hospital, Lahore. Demographic information (name, age, gender, contact details) was also obtained were included in this study. All the patients underwent ESS and were followed up post operatively at the end of $1 \mathrm{st}, 2 \mathrm{nd}, 3 \mathrm{rd}$ and 4 th weeks respectively, to observe the formation of synechiae.

Results: Age range in this study was between 21-40 years out of which a small number of patients were $<20$ years old. Mean age of the patients was $35.30 \pm 10.54$ years. Male patients were $80(53.3 \%)$ while female patients were 70 (46.7\%). Intranasal Synechiae formation was observed in 28 patients $(18.7 \%)$.

Conclusion: Intranasal Synechiae formation between lateral nasal wall and nasal septum was found in $18.4 \%$ of the patients after endoscopic sinus surgery.

Keywords: Sino nasal polyposis, ESS, Intranasal Synechiae Formation
\end{abstract}

\section{Introduction:}

$\mathrm{N}$ asal polyps are presence of an abnormal pedunculated lesions that arise from the diseasedmucosa of paranasal sinuses which later manifest themselves in the nasal cavity. They commonly have solid and cystic components ${ }^{1}, 4-6 \%$ of the general population has Sino nasal polyps. ${ }^{2}$

Sino nasal polyposis is thought to be a multifactorial disease of the nasal mucosa, which is considered clinically by the presence of non-neoplastic translucent swellings in the nasal cavity and paranasal sinuses. It is found in a varied number of diseases and has various histological components that is deter-

\begin{tabular}{|c|c|c|}
\hline Damish Arsalan & & Sarwat Hassan Syed \\
\hline 3. Ghulam Murtaza & & Mohammad Qamar Nasir \\
\hline 5. Muhammad Awais & $\min$ & Amir Ayub Awan \\
\hline 1. ENT, Fatima Mem & ial Hospital, L & ahore \\
\hline 2,4. ENT-I, SIMS, Serv & es Hospital, L & hore \\
\hline 3,5. ENT-II, SIMS, Ser & ces Hospital, I & ahore \\
\hline 6. PGM, Lahore Gen & l Hospital Lal & ore \\
\hline Correspondence: & & \\
\hline Dr. Sarwat Hassan Syec & & \\
\hline ENT-I, SIMS, Services & ospital, Lahor & , sarwathsyed1278@gmail.com \\
\hline Submission Date: & $10-05-2020$ & \\
\hline 1st Revision Date: & $19-09-2020$ & \\
\hline Acceptance Date: & $18-10-2020$ & \\
\hline
\end{tabular}

mined by the underlying disease state. Henceforth, it may embody a common pathological end point innumerous disease processes. ${ }^{3}$

The predominance of nasal polyps in the general populace remains uncertain. However, the reported incidence seems to differ between $0.2 \%$ and $4.3 \%$ of the population. ${ }^{3-5}$ A study conducted in Denmark that lasted over a 6-year period by Larsen and Tos, identified 252 nasal polyposis patients ( 78 females and 174 males) to study its prevalence in the background population of 223449 occupants. Larsen and Tos projected an occurrence of 0.627 per thousand per year in Denmark. ${ }^{6}$

Klossek et al described an overall frequency of $2.11 \%$ in France. ${ }^{7}$ In their study, 10033 subjects were scanned for presence of nasal polyps by use of anauthenticated and structured questionnaire. There is an over-all agreement amongst ENT clinicians that the prevalence increases with rising age and that there is a strong male predominance. ${ }^{6,7}$ Remarkably, a far greater incidence of nasal polyps were reported when autopsy specimens were examined, with nasal polyps 
found in $26-42 \% 8,9$. It is probable that small sized or sessile polyps rarely manifest themselves clinically in the nasal cavity during life. Polyps normally remain asymptomatic, until they progress to such a size as to obstruct the opening of the sinus ostia with subsequent development of symptoms of unremitting nasal obstruction and rhino sinusitis.

There are many disorders that have a recognized incidence of nasal polyps. The reported frequency of nasal polyps in asthmatic patients varies between $6.7 \%$, and $13 \%{ }^{10}$ respectively while the incidence of asthma in patients with Sino nasal polyps has been stated as high as $45 \%{ }^{11}$ In 1968 , Samter defined the Aspirin triad, consisting of Aspirin sensitivity, Atopic Asthma and Sino nasal polyposis. ${ }^{12}$ Hypersensitivity to Aspirin is present in up to $5 \%$ to $10 \%{ }^{13}$ of asthmatic patients and the disease manifestation in such patients is particularly severe. The incidence of nasal polyposis is also higher in the Aspirin tolerant and asthmatic population. The documented prevalence of nasal polyps in this group of patients ranges from $36 \%$ to $95 \%$ respectively. ${ }^{14}$

There are a number of important clinical associations in patients with Sino nasal polyposis, namely CRS, Cystic Fibrosis which should be considered in children with nasal polyps, although the diagnosis will have normally been made in early childhood and Allergic Fungal Rhino sinusitis. The association of nasal polyposis in AFRS is not completely understood. AFRS is associated with thick eosinophilic mucus, lack of fungal invasion into tissues and Type I Hypersensitivity to fungi. The condition is also characterized by bony erosions of the sinus walls seen on CTimaging. ${ }^{15}$

Etiology is still uncertain, chronic inflammatory changes mediated by eosinophils and to some lesser extent neutrophils. Atopy is also implicated in its pathogenesis. ${ }^{2}$ Mainstay of treatment is medical and the most effective treatment of nasal polyps is with topical nasalcorticosteroids and management of the underlying allergy. ${ }^{1,2}$ Patients recalcitrant to medical therapy are treated with endoscopic sinus surgery. ${ }^{16}$

Endoscopic sinus surgery (ESS) is an intranasal surgical procedure, that is performed under direct vision which is negligibly invasive with remarkable improvement of symptoms. Adhesions form due to medialization of the middle and inferior turbinates on the lateral nasal wall and crusting with scarring which results in blockade of the middle meatus and recurrent rhino sinusitis, dictating further surgery. ${ }^{16}$ Factors promoting synechiae formation include history of previous nasal surgery and inexperienced surgical techniques. ${ }^{16}$

Although ESS is considered an extraordinary advancement in nasal surgery, intranasal adhesion formation is an inevitable accompaniment which causes recurrence of disease and dictates a revision surgery. In a literature review by Shamoon et alvariable results of synechiae formation after ESS were reported which concluded that $16.6 \%$ synechiae formation occurred after ESS in a study conducted on 60 patients. ${ }^{17}$ Baradaranfar et al concluded $32.4 \%$ synechiae formation after ESS in a study done on 37 patients. ${ }^{18}$

Intranasal adhesion formation is a distressing complication of ESS. Previous studies conducted were on smaller sample sizes i.e. $37^{18}, 60^{17}$ cases, and we wanted to conduct this study on a larger sample size i.e. 150 cases. Literature showed variable and controversial results. As this surgical procedure is rapidly gaining popularity in our country and no such study has ever been carried out in our set up, so this research has determined the actual rate of intranasal synechiae formation after ESS. The results of this study helped us in determining whether this procedure should be continued in future or some reforms in the surgical technique is needed.

\section{Methods}

This was a descriptive case series study carried out in the department of ENT Unit I, Services Hospital, Lahore, from 03.10.2014 to 02.04.2015. The calculated sample size was 150 cases with $6 \%$ margin of error, $94 \%$ confidence level taking expected percentage of synechiae formation between lateral nasal wall and nasal septum i.e. 16.6\%. Non-probability consecutive sampling was used.

Study cases between age of 16-60 years of both genders were recruited according to the following criteria. All patients with nasal polyps as confirmed on physical examination and computed-tomography (CT). These patients were examined by the ENT consultants in the post-operative follow-up visits and 
labelled as positive for synechiae formation between lateral and medial nasal walls, 4 weeks after ESS, were included in the study. Patients having recurrent nasal polyps with intracranial and intraorbital extension, having previous history of surgery for any nasal pathology and patients with a histologically known Sino nasal malignancy were excluded from the study.

After ethical approval and obtaining written informed consent from 150 patients presenting to ENT Outpatient department of Services Hospital Lahore. A proforma was formulated to record the demographic data for research purpose. All the patients that were treated by ESS were followed up post operatively at the end of $1^{\text {st }}, 2^{\text {nd }}, 3^{\text {rd }}$ and $4^{\text {th }}$ week respectively, and observed for the development of synechiae formation. The patients were categorized as Yes or No for synechiae formation in the Proforma.

\section{Statistical Analysis}

All the collected data was entered into SPSS version 10 and analyzed. The qualitative data like demographics (sex, male or female) and presence of synechiae formation (yes or no) was presented as frequency distribution. Quantitative data like age (in years) was presented as mean and standard deviation.

\section{Results}

Majority of the patients in this study were between 21-40 years of age and minority of the patients were $<$ 20 years old. Mean age of the patients was $35.30 \pm$ 10.54 years (Table- 1$)$.

Male patients were $80(53.3 \%)$ while female patients were $70(46.7 \%)$ in number respectively.

Synechiae formation was observed in 28 patients (18.7\%) (Table-2). Stratification with regard to age and sex was carried out in tables $3 \& 4$ respectively.

\section{Discussion}

Table 1: Distribution of Cases by Age

\begin{tabular}{ccc}
\hline Age (Year) & Number & Percentage \\
\hline$\leq 20$ & 12 & 08.0 \\
$21-40$ & 93 & 62.0 \\
$41-60$ & 45 & 30.0 \\
Total & $\mathbf{1 5 0}$ & $\mathbf{1 0 0 . 0}$ \\
Mean \pm SD & \multicolumn{2}{c}{$\mathbf{3 5 . 3 0} \pm \mathbf{1 0 . 5 4}$} \\
\hline
\end{tabular}

Table 2: Distribution of Cases by Synechiae Formation

\begin{tabular}{ccc}
\hline Synechiae formation & Number & Percentage \\
\hline Yes & 28 & 18.7 \\
No & 122 & 81.3 \\
Total & $\mathbf{1 5 0}$ & $\mathbf{1 0 0 . 0}$ \\
\hline
\end{tabular}

Table 3: Stratification of Age with Regard to Synechiae Formation

\begin{tabular}{cccc}
\multirow{2}{*}{ Age (Year) } & \multicolumn{2}{c}{ Synechiae formation } & Total \\
\cline { 2 - 2 }$\leq 20$ & Yes & No & \\
$21-40$ & 04 & 08 & 12 \\
$41-60$ & 15 & 78 & 93 \\
Total & 09 & 36 & 45 \\
& $\mathbf{2 8}$ & $\mathbf{1 2 2}$ & $\mathbf{1 5 0}$ \\
\hline
\end{tabular}

Table 3: Stratification of Age with Regard to Synechiae Formation

\begin{tabular}{cccc} 
& \multicolumn{2}{c}{ Synechiae formation } & \\
\cline { 2 - 3 } Gender & Yes & No & \\
Male & 13 & 67 & 80 \\
Female & 15 & 55 & 70 \\
Total & $\mathbf{2 8}$ & $\mathbf{1 2 2}$ & $\mathbf{1 5 0}$ \\
\hline
\end{tabular}

Nasal polyps remain a significant challenge to the treat for an otorhinolaryngologist. Association between nasal polyps and chronic rhino sinusitis is unclear but nasal polyps should be regarded as an accompaniment of chronic inflammation of the Sino nasal mucosa. ${ }^{19}$

The management of nasal polyps includes both medical and surgical options following the evaluation of the patient, but main stay of treatment is medical. Surgery alone cannot accomplish or sustain healthy Sino nasal mucosa. In surgery, unhealthy mucosa is removed that did not initially respond to preoperative medical therapy. Surgery may be able to reestablish mucociliary clearance, remove diseased mucosa, refining sinus ventilation and permitting access to topical nasal corticosteroids, but surgery alone cannot alleviate Sino nasal disease. Patients need to be encouraged to continue persistent medical treatment postoperatively in order to achieve unsurpassed results which may need prolonged medical management after surgery with regular follow up visits.

Surgical treatment encompasses intranasal polypectomy which has an extraordinary recurrence rate, intranasal ethmoidectomy - a blind procedure that also results in complications like reappearance of disease and external ethmoidectomy, with its charac- 
teristic complication of an external scar. All these limitations are surpassed by the progression of ESS, which is fast becoming afetching surgical treatment of choice for Sino nasal polyposis. ${ }^{20}$

Endoscopic sinus surgery (ESS) has become the customary treatment of choice for the management of medically recalcitrant chronic rhino sinusitis and nasal polyps. The most common complication ofESS is postoperative synechiae formation with a prevalence ranging from $1 \%$ to $36 \%{ }^{21}$ respectively. Retained secretions, mucosal damage, scar formation, and excessive nasal crusting aids in intranasal synechiae formation. Adhesions can impede the normal mucociliary clearance of the sinuses and results in a relapse. Numerous surgical practices like partial resection of the middle turbinate, and placement of intranasal biodegradable packing materials, have helped to lessen the incidence of postoperative synechiae formation. ${ }^{22}$ Conventional nasal packing agents, such as Vaseline gauze strips and expandable polyvinyl acetate (Merocel) are non-absorbable and new biodegradable packing materials with various grades of effectiveness have also been developed, for example, Floseal, MeroGel/Meropak, Nasopore and carboxymethylcellulose. ${ }^{23}$ Use of Mitomycin C during surgery has also helped decrease Synechiae formation after $\mathrm{ESS}^{24}$

Largely, the incidence of intranasal synechiae formation after ESS varies significantly from trial to trial, and the ideal material for nasal packing is still a matter of debate.

In the present study, majority of the patients 93 (62\%) in the age group of 21-40 years who underwent ESS, were consistent with the findings of other studies $(70 \%){ }^{25-27}$

In the existing study, intranasal synechiae formation after endoscopic sinus surgery was observed in $18.7 \%$ of the patients. Our findings are similar with the study carried out by Shumon et al. ${ }^{17}$ Baradaranfar et al differ with our findings, as they confirmed $32.4 \%$ synechiae formation after ESS. ${ }^{18}$

\section{Conclusion}

Intranasal Synechiae formation was established in $18.4 \%$ of the patients who were subjected to endoscopic sinus surgery. Endoscopic sinus surgery has delivered a safe and effective method for dealing with various Sino nasal diseases. Most frequently, this procedure can be carefully and effectively done under a local anesthesia. Rigid nasal endoscope offers a well-lit view into the nasal cavity and the osteomeatal complex which is the cornerstone of ESS, so that Sino nasal illnesses can be faredwell with a high rate of success and improvement of symptoms with trivial morbidity. Postoperative follow up visits are as important as surgery that should be personalized according to the patient's requirements.

\section{Author's Contributions \\ DA: Data Collection \\ SHS: Introduction, literature review, Disscussion \\ GM,AAA: Article references \\ MQN: StatisticalAnalysis \\ MAA: Edited}

\section{References}

1. Aydil U, Karadeniz H, Sahin C. Choanal polyp originated from the inferior nasal concha. Eur Arch Otorhinolaryngol 2008;265:477-9.

2. Yaman H, Yilmaz S, Karali E, Guclu E, Ozturk O. Evaluation and Management of Antrochoanal Polyps. Clin Exp Otorhinolaryngol 2010;3:110-4.

3. Tos M, Larsen PL. Nasal Polyps: Origin, Etiology, Pathogenesis and Structure. In: Kennedy DW, Bolger WE, Zinreich SJ, editors Diseases of the sinuses: Diagnosis and Management 1st ed Philadelphia: Decker Inc 2001. P. 455-501.

4. Hedman J, Kaprio J, Poussa T, Nieminen MM. Prevalence of asthma, aspirin intolerance and chronic obstructive pulmonary disease in a population based study. Int J Epidemiol 1999;28: 717-22.

5. Mygind N, Dahl R, Bachert C. Nasal polyposis, eosinophils dominated inflammation and allergy. Thorax 2000;55:S79-S83

6. Larsen K, Tos M. The estimated incidence of symptomatic nasal polyps. Acta Otolaryngol 2002; 122: 179-82.

7. Klossek JM, Neukirch F, Pribil C, Jankowski R, Serrano E, Chanal I, El Hasnaoui A. Prevalence of nasal polyposis in France: A cross-sectional, casecontrol study. Allergy 2005;60:233-7.

8. Larsen PL, Tos M. Site of origin of nasal polyps. Transcranially removed naso-ethmoidal blocks as a screening method for nasal polyps in autopsy material. Rhinology 1994;33:185-8.

9. Larsen PL, Tos M. Anatomic site of origin of nasal polyps: endoscopic nasal and paranasal sinus surgery 
as a screening method for nasal polyps in autopsy material. Am J Rhinol 1996;10:211-6.

10. Stierna PLE. Nasal polyps: Relationship to infection and inflammation. Allergy Asthma Proc 1996;17: 251-7.

11. Rugina M, Serrano E, Klossek JM, Crampette L, Stoll D, Begear JP, et al. Epidemiological and clinical aspects of nasal polyposis in France: the ORLI group experience. Rhinology 2002;40:75-9.

12. Samter M, Beers RF. Intolerance to aspirin. Clinical studies and consideration of its pathogenesis. Ann Intern Med 1968;68:975-83.

13. Szczeklik A, Steveson DD. Aspirin induced asthma: advances in pathogenesis and management. J Allergy Clin Immunol 1999; 104:5-13.

14. Larsen $\mathrm{K}$. The clinical relationship of nasal polyps to asthma. Allergy Asthma Proc 1996;17:243-9.

15. Hadfield PJ, Rowe Jones JM, Mackay IS. The prevalence of nasal polyps in adults with cystic fibrosis. Clin Otol 2000;25:19-22.

16. Eitan Y, Jacob S, Tamara D, Rami T, Tuvia H. Recurrence of nasal polyps after functional endoscopic sinus surgery. Conexiuni Med 2009;4: 27-9.

17. Shumon AM, Zaman KA, Hossain M, Zaman Y, Azad A. Nasal polypectomy and endoscopic sinus surgery for the management of ethmoidal polyp. Bangladesh J Otorhinolaryngol 2012;18:161-5.

18. Baradaranfar MH, Khadem J, Taghipoor Zahir S, Kouhi A, Dadgarnia MH, Baradarnfar Prevention of adhesion after endoscopic sinus surgery: role of mitomycin C. A. Acta Med Iran 2011;49:131-5.

19. European Academy of Allergology and Clinical Immunology. European position paper on rhino sinusitis and nasal polyps. EAACI Task Force. Rhinology Supplement 2005;18:1-87

20. Kirtane MV, Patel H, Muranjan S. Microdebrider in endoscopic sinus surgery. In: challenges in otolaryngology, 1s' ed. Jaypee Brothers 2005. P. 4142.

21. Anand VK, Tabaee A, Kacker A, Newman JG, Huang C. The role of mitomycin $\mathrm{C}$ in preventing synechia and stenosis after endoscopic sinus surgery. Am J Rhinol 2004; 18:311-4.

22. Catalano PJ, Roffman EJ. Evaluation of middle meatal stenting after minimally invasive sinus techniques (MIST). Otolaryngol Head Neck Surg 2003; 128:875-81.

23. Weber R, Keerl R, Hochapfel F, Draf W, Toffel PH. Packing in endonasal surgery. Am J Otolaryngol 2001;22:30620.

24. Chung JH, Cosenza MJ, Rahbar R, Metson RB. Mitomycin $\mathrm{C}$ for the prevention of adhesion formation after endoscopic sinus surgery: a randomized, controlled study. Otolaryngol Head Neck Surg 2002;126:468-74.

25. Stammberger H. Endoscopic endonasal surgery concepts in treatment of recurring rhinosinusitis. Part II - Surgical technique. Otolaryngol Head Neck Surg 1986;94:147-56.

26. Lund Valerie J. Nasal Polyps: Epidemiology, pathogenesis and treatment, Ocean Side Publications Inc 2001.

27. Alam M. Functional endoscopic sinus surgery (FESS) - Our experience at BSMMU. Bangladesh J Otorhinolaryngol 2003;4:11-14. 